\title{
Optimal bundle pricing with monotonicity constraint.
}

Citation for published version (APA):

Grigoriev, A., van Loon, J., Sviridenko, M., Uetz, M. J., \& Vredeveld, T. (2008). Optimal bundle pricing with monotonicity constraint. METEOR, Maastricht University School of Business and Economics. METEOR Research Memorandum No. 015 https://doi.org/10.26481/umamet.2008015

Document status and date:

Published: 01/01/2008

DOI:

10.26481/umamet.2008015

Document Version:

Publisher's PDF, also known as Version of record

\section{Please check the document version of this publication:}

- A submitted manuscript is the version of the article upon submission and before peer-review. There can be important differences between the submitted version and the official published version of record.

People interested in the research are advised to contact the author for the final version of the publication, or visit the DOI to the publisher's website.

- The final author version and the galley proof are versions of the publication after peer review.

- The final published version features the final layout of the paper including the volume, issue and page numbers.

Link to publication

\footnotetext{
General rights rights.

- You may freely distribute the URL identifying the publication in the public portal. please follow below link for the End User Agreement:

www.umlib.nl/taverne-license

Take down policy

If you believe that this document breaches copyright please contact us at:

repository@maastrichtuniversity.nl

providing details and we will investigate your claim.
}

Copyright and moral rights for the publications made accessible in the public portal are retained by the authors and/or other copyright owners and it is a condition of accessing publications that users recognise and abide by the legal requirements associated with these

- Users may download and print one copy of any publication from the public portal for the purpose of private study or research.

- You may not further distribute the material or use it for any profit-making activity or commercial gain

If the publication is distributed under the terms of Article $25 \mathrm{fa}$ of the Dutch Copyright Act, indicated by the "Taverne" license above, 
Alexander Grigoriev, Joyce van Loon, Maxim Sviridenko, Marc Uetz, Tjark Vredeveld

Optimal Bundle Pricing with Monotonicity Constraint

$\mathrm{RM} / 08 / 015$

JEL code: C44, C61

\section{METEबrR}

Maastricht research school of Economics of TEchnology and ORganizations

Universiteit Maastricht

Faculty of Economics and Business Administration P.O. Box 616

NL - 6200 MD Maastricht

phone : ++31433883830

fax : ++31433884873 


\title{
Optimal Bundle Pricing with Monotonicity Constraint
}

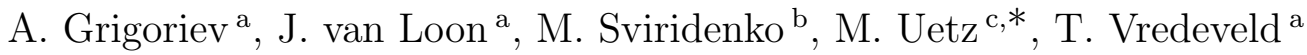 \\ ${ }^{a}$ Maastricht University, Quantitative Economics, \\ P.O.Box 616, 6200 MD Maastricht, The Netherlands \\ b IBM T.J. Watson Research Center, \\ P.O. Box 218, Yorktown Heigths, NY 10598, USA \\ ${ }^{\mathrm{c}}$ University of Twente, Applied Mathematics, \\ P.O. Box 217, 7500 AE Enschede, The Netherlands
}

\begin{abstract}
We consider the problem to price (digital) items in order to maximize the revenue obtainable from a set of bidders. We suggest a natural monotonicity constraint on bundle prices, show that the problem remains NP-hard, and we derive a PTAS. We also discuss a special case, the highway pricing problem.
\end{abstract}

Key words: bundle pricing, revenue optimization, monotonicity, computational complexity, approximation algorithm

\section{Introduction}

We consider the situation where we want to sell a set of (digital) items to a set of bidders. Every bidder places bids on subsets, or bundles of items, and each bidder would like to receive one or more of these bundles (OR-bids). Bidders have valuations for each of the bundles they bid on. The valuation is the maximum amount a bidder is willing to pay for a particular bundle. We assume that the bundles and the valuations are known. Hence we are faced with a purely algorithmic problem, in contrast to mechanism design problems where the valuations are private information to the bidders. We assume that each item is available in unlimited supply, as for example for digital goods. We address a standard pricing regime often addressed in the literature, namely each item needs to receive an anonymous price, and the price of any bundle is the sum of its item prices. The optimization problem is to determine single item prices so as to maximize the total revenue collected from all bidders. Notice that the problem is nontrivial only because not all bids need to be granted in an optimal solution, as otherwise the problem reduces to solving a linear program. Also notice that any solution will be trivially envy-free, meaning that a bidder not receiving a particular bundle cannot afford it. This is an artifact of the unlimited supply of items.

In a sequence of recent papers $[1,2,5,7,10,12,13]$, several algorithms and complexity results have been derived for such price optimization problems. Many of the positive results achieve only logarithmic approximation guarantees, and often somewhat trivial algorithms suffice to achieve such bounds, e.g. uniform prices

\footnotetext{
* Corresponding author.

Email addresses: a.grigoriev@ke.unimaas.nl (A. Grigoriev), j.vanloon@ke.unimaas.nl (J. van Loon), sviri@us.ibm.com (M. Sviridenko), m.uetz@utwente.nl (M. Uetz), t.vredeveld@ke.unimaas.nl (T. Vredeveld).
} 
for all items. Demaine et al. [6] delivered a reason for this observation on the performance of algorithms, showing that -in general- the problem does not allow for semi-logarithmic approximation algorithms. In this paper, we introduce a very natural monotonicity constraint on bundle prices that allows us to derive results that break this semi-logarithmic inapproximability barrier. More specifically, we impose the condition that the price of any bundle of size $k$ must not exceed the price of a bundle of size $k+1$ or larger, for any $k$. As we will show, this condition implies that most of the items are comparable in the sense that their prices cannot differ too much. Before we elaborate on related work and our contribution in more detail, let us define the pricing settings more formally.

\subsection{Model}

Let $I=\{1, \ldots, m\}$ denote the set of items for sale, and let $J=\{1, \ldots, n\}$ denote the set of bids placed by all bidders. Each bid $j \in J$ is on exactly one subset of items $I_{j} \subseteq I$. In line with notation in auction literature, we call the set $I_{j}$ also a bundle. Every bidder has a positive valuation for each of her bundles, that is, every bundle $I_{j}$ corresponding to bid $j \in J$ has a positive valuation $b_{j}$ which is the maximum amount its bidder is willing to pay for bundle $I_{j}$. We may assume without loss of generality that $b_{j} \geq 1$ for $j \in J$. By $p_{i}$ we denote the price of item $i$, for $i=1, \ldots, m$. The price of a bundle $I_{j}$ is therefore $\sum_{i \in I_{j}} p_{i}$. A bid is a winning bid if its bidder can afford it, and a losing bid otherwise. The set of winning bids is denoted by $W \subseteq J$.

Definition 1 Given are $n$ bids of the form $\left(I_{j}, b_{j}\right), j \in J$. Each bid $j$ has valuation $b_{j}$ and is placed on a bundle $I_{j} \subseteq I$, where $I$ is a set of $m$ items of unlimited availability each. A solution consists of a price $p_{i}$ for every item $i \in I$. Given item prices, let $W \subseteq J$ denote all winning bids, so $\sum_{i \in I_{j}} p_{i} \leq b_{j}$ for all $j \in W$ and $\sum_{i \in I_{j}} p_{i}>b_{j}$ for all $j \in J \backslash W$. The objective is to maximize the total revenue $\sum_{j \in W} \sum_{i \in I_{j}} p_{i}$.

This problem is in general not approximable to within a (semi-)logarithmic factor in the number of bids $n$ [6]. We therefore introduce a monotonicity constraint on the set of feasible price vectors. More specifically, we impose the following condition for any two subsets of items $I^{\prime}$ and $I^{\prime \prime}$.

$$
\sum_{i \in I^{\prime}} p_{i} \leq \sum_{i \in I^{\prime \prime}} p_{i} \text { whenever }\left|I^{\prime}\right|<\left|I^{\prime \prime}\right|
$$

The condition has a meaningful economic interpretation in settings where items are different, yet comparable. It only requires that larger bundles are at least as expensive as smaller ones. Notice that we do not require a monotonicity condition on bundles of the same size, as this would immediately yield that all items must receive the same price. Yet, the condition is quite strong, as it requires that (most) item prices are of the same order of magnitude; see Lemma 6 . We show how this monotonicity constraint can be exploited to derive results that break the inapproximability barrier known for the general case.

In the remainder of this paper, we denote by a $\rho$-approximation algorithm an algorithm that produces a solution with value at least $1 / \rho$ times the optimal solution value. A PTAS is a family of polynomial time $(1+\varepsilon)$-approximation algorithms, for any $\varepsilon>0$.

\subsection{Related work}

Pricing problems with unlimited availability of items and single item pricing regime have recently received quite some attention in the algorithmic literature $[1,2,5-7,10-13]$. As mentioned earlier, in general the maximum revenue is hard to approximate to within a semi-logarithmic factor in the number of bids $n$ [6]. In particular, it is therefore unlikely that a constant approximation algorithm exists. For the same problem, Hartline and Koltun [13] present an approximation scheme with almost linear running time, given that the number of distinct items $m$ is constant. Moreover, given that each bidder is interested in bundles of at most $k$ items, Balcan and Blum [2] derive an $\mathrm{O}(k)$-approximation. Finally, there exist two fully polynomial time approximation schemes $[2,5]$ for the problem where the bundles are a laminar family, that is, for any two bundles $I_{j}$ and $I_{k}$ it holds that $I_{j} \subseteq I_{k}, I_{k} \subseteq I_{j}$ or $I_{j} \cap I_{k}=\emptyset$. 
Notice that an instance where bundles are a laminar family can always be interpreted as a problem with a linear order on the items, and where the bundles consist of consecutive items in that linear order. The latter problem, but now without the requirement that bundles are a laminar family, is known as the "highway problem" [12]. Even this seemingly innocent problem is NP-complete [5]. The highway problem is motivated by the problem to determine tolls to be charged for usage of parts of a highway. For that problem, there exists an $\mathrm{O}(\log m)$-approximation [2], and given that the largest valuation is bounded by a constant, also an exact dynamic programming algorithm [12].

\subsection{Our results}

We show in Section 2 that the revenue maximization problem of Definition 1 is strongly NP-hard even with the monotonicity constraint on bundle prices as defined in (1). Moreover, we derive a polynomial time approximation scheme (PTAS) for that problem, with a time complexity of $\left.\mathrm{O}\left(n m^{8 / \varepsilon}(\log B)\right)^{8 / \varepsilon}\right)$, where $B=\max _{j} b_{j}$. The PTAS crucially exploits the fact that the monotonicity condition (1) yields most item prices to be of the same order of magnitude.

As a special case we also address the "highway problem" as suggested in [12] in Section 3. We show that also this problem remains weakly NP-hard under the monotonicity condition, and we derive a simple $\mathrm{O}(\log B)$-approximation algorithm, where $B=\max _{j \in J} b_{j}$.

\section{Arbitrary Bundles}

We settle the computational complexity of the pricing problem with monotonicity constraint by first showing strong NP-hardness, and then deriving a PTAS.

\subsection{Complexity}

We show that the pricing problem with monotonicity constraint is strongly NP-hard by using a reduction from the strongly NP-hard problem IndePendetSet [8]. Let $G=(V, E)$ be a graph in which we want to find a maximum cardinality set of vertices that are pairwise non-adjacent. We define an instance $\mathcal{I}$ of the pricing problem as follows. Let $M$ be an integer that is large enough. For every vertex $v \in V$ we create a vertex-item, and for every edge $e \in E$ we introduce an edge-item, that is, $I=V \cup E$. For every item $i \in I$, there are $M+2$ bids placed on the bundle consisting of only this item. One of these bids has valuation $M$, and the others have the same valuation $M+1$. Moreover, for every edge $e=\{u, v\} \in E$, there are four more bids. One bid is on bundle $\{u, e\}$, one bid on bundle $\{v, e\}$, and two bids are on bundle $\{u, v\}$. These four bids each have valuation $2 M+1$ each. Here, $M$ is an integer large enough, for example, $M \geq 2|E|^{2}+4|E|+2$ suffices.

In the NP-hardness proof (Theorem 5) we need the property that in an optimal solution to the above created instance $\mathcal{I}$ of the pricing problem all prices are either $M$ or $M+1$. Therefore, we first collect some preliminary observations.

Lemma 2 In the optimal solution to the pricing problem on $\mathcal{I}$, all prices are within the interval $[M, M+1]$.

PROOF. Assume the claim is not true, and let $p$ be an optimal solution that violates it. If all items have prices $p_{i} \leq M+1$ and there exists at least one item with price $p_{i}<M$, then it can easily be verified that the price vector $p^{\prime}$, defined by $p_{i}^{\prime}=\max \left\{p_{i}, M\right\}$, yields the same set of winning bids and the revenue has increased compared to $p$. Hence, $p$ cannot be optimal.

Therefore, assume that at least one item, $h$, has a price $p_{h}>M+1$ and consider the price vector $p^{\prime}$ defined by $p_{i}^{\prime}=p_{i}$ for $i \neq h$ and $p_{h}^{\prime}=M+1$. As item $h$ belongs to at most $3|E|$ bundles requesting two items, the decrease in revenue of the two item-bundles due to the price change is at most $3|E| M$. On the other hand, $M+1$ bidders can now afford their bundle requesting item $h$. Therefore, the increase in revenue 
due to these bidders is $M^{2}+2 M+1$. Thus, the total revenue increases by at least $M^{2}+(2-3|E|) M+1$ which is positive since $M>2|E|^{2}$. Hence, the price vector $p$ cannot be optimal.

Lemma 3 In an optimal solution to the pricing problem on $\mathcal{I}$, all bids on two-item bundles are winning bids.

PROOF. Suppose that there exists a two-item bundle, say $I_{j}=\left\{i_{1}, i_{2}\right\}$, that is priced higher than $2 M+1$ and let the prices of the two items be $p_{i_{1}}=M+d_{1}$ and $p_{i_{2}}=M+d_{2}$. By the previous lemma, we know that $0<d_{1}, d_{2} \leq 1$. As each item is in at most $3|E|$ two-item bundles, decreasing the price of item $i_{1}$ to $M$ increases the total revenue by at least $-((M+1)+(3|E|-1)) d_{1}+M+2 M+d_{2} \geq 2 M-3|E|$, which is positive since $M>3|E| / 2$.

Lemma 4 In an optimal solution to the pricing problem on $\mathcal{I}$, all prices are integral.

PROOF. By Lemma 2 we know that each item has a price $p_{i}=M+d_{i}$, for $0 \leq d_{i} \leq 1$. Suppose that there exists an item $i$ with $0<d_{i}<1$ and that this item corresponds to an edge $e=\{u, v\}$. If both $d_{u}=d_{v}=0$, then we can change the price of the edge item $e$ to $p_{e}^{\prime}=M+1$ without affecting the set of winning bids and increasing the total revenue. Therefore, assume that $d_{u}>0$ and that $d_{u} \geq d_{v}$. By Lemma 3 , we know that $d_{u}+d_{v} \leq 1$ and thus $d_{v} \leq 1 / 2$. Consider the prices $p^{\prime}$ defined by $p_{e}^{\prime}=M+1, p_{u}^{\prime}=p_{v}^{\prime}=M$, and $p_{i}^{\prime}=p_{i}$ for all other items $i$. The increase in revenue due to this price change for the two-item bundles corresponding to the edge $e=\{u, v\}$ is $1-\left(d_{u}+d_{e}\right)+1-\left(d_{v}+d_{e}\right)-2\left(d_{u}+d_{v}\right)$, whereas the total decrease in revenue for all other two-item bundles is bounded by $3(|E|-1)\left(d_{u}+d_{v}\right)$. The increase in revenue caused by the single item bundles $e$ and $u$ is $\left(1-d_{e}\right)(M+1)+M-d_{u}(M+1)$, whereas the increase in revenue for the single item bundles on $v$ is $M-(M+1) d_{v} \geq 0$ if $d_{v}>0$ and 0 otherwise. Hence, the total increase in revenue due to this price change is $1-\left(d_{u}+d_{e}\right)+1-\left(d_{v}+d_{e}\right)-2\left(d_{u}+d_{v}\right)-3(|E|-1)\left(d_{u}+d_{v}\right)+\left(1-d_{e}\right)(M+1)+M-d_{u}(M+1) \geq$ $M-(3|E|-1)\left(d_{u}+d_{v}\right)+\left(1-d_{e}-d_{u}\right)(M+1) \geq M-(3|E|-1) \geq 0$, as $M>3|E|-1$. Hence, any solution in which an edge item $e$ has a fractional price cannot be optimal.

On the other hand, suppose that all edge items $e \in E$ have price $p_{e} \in\{M, M+1\}$ and there exists a vertex item $u$ with $M<p_{u}<M+1$. Then by Lemma 3 we know that there exists an item $u$ such that all its neighbors $v$ with $\{u, v\} \in E$ have price $p_{v} \leq M+1 / 2$. Moreover, we may assume that there exists a neighbor $v$ such that $p_{v}>M$ as otherwise we may set $p_{u}=M+1$ without affecting the set of winning bids, but increasing the total revenue. Let $d=\max \left\{d_{v}:\{u, v\} \in E\right\}$, then $0<d \leq 1 / 2$. Consider the prices $p^{\prime}$ defined by $p_{u}^{\prime}=M+1, p_{v}^{\prime}=M$ for $v$ such that $\{u, v\} \in E$ and $p_{i}^{\prime}=p_{i}$ for all other items $i$. Then the revenue for each two-item bundle is decreased by at most $2 d$ and as there are in total $4|E|$ two-item bundles the total decrease in revenue due to this price change is bounded by $4|E|(2 d) \leq 4|E|$. As there was at least one neighbor of $u$ that had a price $p_{v}>M$, the change in price leads to an increase of revenue of at least $\left(1-d_{u}\right)(M+1)+M-d(M+1) \geq\left(1-d_{u}-d\right)(M+1)+M \geq M$, as by Lemma $3 d_{u}+d \leq 1$. Hence the total increase in revenue due to this price change is at least $M-4|E|>0$, as $M>4|E|$. Hence, no solution with at least one fractional price can be optimal.

Theorem 5 The revenue maximization problem is strongly NP-hard, even if the prices need to satisfy the monotonicity constraint.

PROOF. Consider instance $\mathcal{I}$. We claim that there exists an independent set of size $s$ in $G$ if and only if there is a solution to the revenue maximization problem with revenue at least $f(s):=\left(M^{2}+2 M\right)|V|+$ $\left(M^{2}+10 M+3\right)|E|+s$.

Given an IndePendetSet $V^{\prime} \subseteq V$ with $\left|V^{\prime}\right|=s$. Let $E_{0}=\left\{e=\{u, v\} \in E: u, v \notin V^{\prime}\right\}$. Let $p_{v}=M+1$ if $v \in V^{\prime}$ and $M$ otherwise. Let $p_{e}=M+1$ if $e \in E_{0}$ and $M$ otherwise. The revenue of this solution is $\pi=(M+1)\left(M|V|+\left|V^{\prime}\right|+M|E|+\left|E_{0}\right|\right)+M\left|V \backslash V^{\prime}\right|+M\left|E \backslash E_{0}\right|+8 M|E|+2\left|E_{0}\right|+3\left|E \backslash E_{0}\right|=$ $\left(M^{2}+2 M\right)|V|+\left(M^{2}+10 M+3\right)|E|+\left|V^{\prime}\right|=f(s)$. 
For the converse, assume there is an optimal solution of the pricing problem with revenue at least $f(s)$. We know by Lemmas 2 and 4, that the price for any item is either $M$ or $M+1$. Let $V^{\prime}=\{v \in V$ : $\left.p_{v}=M+1\right\}$, then $V^{\prime}$ is an independent set in $G$, as by Lemma 3 we know that for any $e=\{u, v\} \in E$ with $u \in V^{\prime}, p_{v} \leq M$. Define $E_{0}=\left\{e=\{u, v\} \in E: p_{e}=M+1\right\}$. Then for any $e=\{u, v\} \in E_{0}$, we have that $p_{u}=p_{v} \leq M$ by Lemma 3. As the revenue for the four two-item bundles corresponding to an edge $e \in E \backslash E_{0}$ is bounded by $3(2 M+1)+2 M=8 M+3$ the total revenue is bounded from above by $\left|V^{\prime}\right|(M+1)^{2}+\left|V \backslash V^{\prime}\right|\left(M^{2}+2 M\right)+\left|E_{0}\right|(M+1)^{2}+\left|E \backslash E_{0}\right|\left(M^{2}+2 M\right)+\left|E_{0}\right|(2(2 M+1)+2(2 M))+\mid E \backslash$ $E_{0}\left|(8 M+3)=\left(M^{2}+2 M\right)\right| V|+| V^{\prime}\left|+\left(M^{2}+10 M+3\right)\right| E \mid$. As the total revenue is at least $f(s)$, we conclude that $\left|V^{\prime}\right| \geq s$, which finishes the proof.

\subsection{Approximation scheme}

Assume, without loss of generality, that $p_{1} \leq p_{2} \leq \ldots \leq p_{m}$, then by the monotonicity constraint, we know $2 p_{2} \geq p_{1}+p_{2} \geq p_{m}$. Similarly, $3 p_{3} \geq p_{1}+p_{2}+p_{3} \geq p_{m-1}+p_{m} \geq 2 p_{m-1}$, etc.

Lemma 6 Suppose $p_{1} \leq p_{2} \leq \ldots \leq p_{m}$. Any pricing of the items satisfying the monotonicity constraint also satisfies

$$
k p_{k} \geq(k-1) p_{m-k+2}, \quad k=2, \ldots,\left\lceil\frac{m}{2}\right\rceil .
$$

The idea for the PTAS is now the following. First, we restrict the prices to powers of $(1+\delta)$ for some $\delta>0$. Then, Lemma 6 says that except for a constant number of the cheapest and most expensive items, all items have prices in roughly the same range. Therefore we can price all except a constant number of items uniformly with the same price, without loosing too much in terms of the total revenue. We therefore enumerate over all possible uniform prices for the bulk of the items, and over all possible combinations of prices for the remaining (constant number of) items.

Theorem 7 The pricing problem with monotonicity constraint admits a PTAS. The time complexity is $O\left(n m^{8 / \varepsilon}(\log B)^{8 / \varepsilon}\right)$, where $\varepsilon$ is the precision of the PTAS and $B=\max _{j} b_{j}$.

PROOF. Given an instance of the pricing problem and an $\varepsilon>0$, let $\delta=\varepsilon / 4$, and for convenience assume that $1 / \delta$ is integral. Let $p_{1} \leq \cdots \leq p_{m}$ be the prices in an optimal solution, satisfying the monotonicity constraints. Define the subsets of items $S=\left\{i \in I: i \leq \frac{1}{\delta}\right\}, M=\left\{i \in I: 1+\frac{1}{\delta} \leq i \leq m+1-\frac{1}{\delta}\right\}$ and $L=\left\{i \in I: i \geq m+2-\frac{1}{\delta}\right\}$. Note that $M=\emptyset$ if $\varepsilon \leq 8 /(m+1)$, in which case the number of items is in $O(1 / \varepsilon)$. We round down the prices of all items in $S$ and $L$ to powers of $(1+\delta)$. Moreover, we price all items in $M$ uniformly at price $p_{1+1 / \delta}$, rounded down to a power of $(1+\delta)$. Let us call the new prices $p^{\prime}$, and let us call $p_{M}^{\prime}$ the price of items in $M$. First observe that the order of prices does not change. We next argue that we do not loose too much by this rounding. Clearly, since we only round down, the set of winning bids can only increase. Moreover, we loose at most a factor $(1+\delta)$ on items in $S$ and $L$. Finally, consider the items in $M$. By (2), we have

$$
\left(1+\frac{1}{\delta}\right) p_{1+1 / \delta} \geq \frac{1}{\delta} p_{m+1-1 / \delta}
$$

In other words, the price for the most expensive item in $M$ differs from the cheapest item in $M$ by a factor at most $(1+\delta)$. Hence, on items in $M$ we loose a factor at most $(1+\delta)^{2}$.

Now we have a structured solution, but it may violate the monotonicity constraint. In case of a violation, we divide the highest prices by a factor of $(1+\delta)$, so that the new price vector satisfies the monotonicity constraint. Note that in this case, we loose at most a factor of $(1+\delta)^{3}$ compared to the optimal pricing vector. The question remains which prices need to be divided by $(1+\delta)$. To this end, we define $s=\max \{0, i$ : $\left.p_{i}^{\prime}<p_{M}^{\prime}\right\}$ as the number of small prices and $\ell=\max \left\{0, i: p_{m+1-i}^{\prime}>p_{M}^{\prime}\right\}$ as the number of high prices. If $s>\ell$, then the new prices are defined by $p_{i}^{\prime \prime}=p_{i}^{\prime}$ for $1 \leq i \leq s$ and for $s+1 \leq i \leq m$ by $p_{i}^{\prime \prime}=p_{i}^{\prime} /(1+\delta)$. Otherwise, if $s \leq \ell$, then the new prices are defined by $p_{i}^{\prime \prime}=p_{i}^{\prime}$ for $1 \leq i \leq m-\ell$ and for $m-\ell+1 \leq i \leq m$ by $p_{i}^{\prime \prime}=p_{i}^{\prime} /(1+\delta)$. Let $k=\max \{s-1, \ell\}$. Then, if there is a violation of the monotonicity constraint in the new 
pricing, sets $\{1, \ldots, k+1\}$ and $\{m+1-k, \ldots, m\}$ are the two most violating sets, as $p_{k+2}^{\prime \prime}=p_{m-k}^{\prime \prime}=p_{M}^{\prime}$. Therefore, we only need to verify that for these two sets the monotonicity constraint holds true. First notice that by the way of defining $k$ and the prices $p^{\prime \prime}$, we have that $p_{i}^{\prime \prime}=p_{i}^{\prime}$ for $1 \leq i \leq k+1$ and $p_{i}^{\prime \prime}=p_{i}^{\prime} /(1+\delta)$, for $m+1-k \leq i \leq m$. Hence, we have that

$$
\sum_{i=1}^{k+1} p_{i}^{\prime \prime}=\sum_{i=1}^{k+1} p_{i}^{\prime} \geq \sum_{i=1}^{k+1} \frac{p_{i}}{1+\delta} \geq \sum_{i=m+1-k}^{m} \frac{p_{i}}{1+\delta} \geq \sum_{i=m+1-k}^{m} \frac{p_{i}^{\prime}}{1+\delta}=\sum_{i=m+1-k}^{m} p_{i}^{\prime \prime},
$$

where the second inequality is due to the monotonicity of the original set of prices $p$. From this it follows that the structured solution $p^{\prime \prime}$ satisfies the monotonicity constraint.

The PTAS now consists of enumerating all possible structured solutions, which is sufficient to obtain a feasible solution that differs from the optimal solution by a factor at most $(1+\delta)^{3}<(1+\varepsilon)$. There are $\left(\begin{array}{c}m \\ -1+2 / \delta\end{array}\right)$ possible choices for $S \cup L$. Since all prices are powers of $(1+\delta)$, there are $\log B$ possible prices. Given that all items in $M$ have the same price, there are at most $(\log B)^{2 / \delta}$ structured solutions for each choice of $S \cup L$. Computation of the revenue for any such solution takes $\mathrm{O}(\mathrm{nm})$ time. This together with $\delta=\varepsilon / 4$ yields the claimed time complexity, where the constant hidden in the $O$-notation depends on $\varepsilon$.

\section{Special case: The highway problem}

A particularly intriguing special case of the general pricing problem considered so far is the "highway problem" as introduced by Guruswami et al. [12]. Here, the items are edges of a simple path, and the bundles corresponding to bids requested by bidders are subpaths. The problem is known to be weakly NP-hard [5], and a $\log (m)$-approximation exists [2]. Recently, also a quasi-PTAS [7] has been derived. The existence of a constant-factor approximation algorithm, however, is still open.

In this setting, it is most natural to assume that the monotonicity constraint holds for any two subpaths only, but not necessarily for arbitrary subsets of items. Hence, the strong NP-hardness result and the PTAS for the revenue maximization problem with monotonicity constraint, established in the previous section, do not carry over to the highway problem with price monotonicity on subpaths. Also notice that the weak NP-hardness for the general highway problem from [5] does not automatically yield weak NP-hardness for the problem with price monotonicity as optimal prices in that completeness proof are not monotone in the length of the subpaths. We next derive weak NP-hardness for the problem with monotonicity constraint, and we present a simple $\mathrm{O}(\log B)$-approximation algorithm, where $B=\max _{j \in J} b_{j}$.

\subsection{Complexity}

We prove NP-completeness of the highway problem with monotonicity constraint by a reduction from EqualCardinalitySubsetSum: Given a set of positive integers $a_{1}, a_{2}, \ldots, a_{2 L}$ and nonnegative integer $A$, does there exist a set $S \subseteq\{1, \ldots, 2 L\}$ such that $\sum_{\ell \in S} a_{\ell}=A$ and $|S|=L$ ? This problem is readily shown NP-complete by a simple reduction from SubSETSum (see [8]).

Consider an instance of EqualCardinalitySubsetSum with $0 \leq a_{1} \leq a_{2} \leq \ldots \leq a_{2 L}$ and assume that $a_{2 L}>\sum_{\ell=1}^{2 L-1} a_{\ell}$ and $0<A<a_{2 L}$. These assumptions do not harm the NP-completeness of the problem, as we can always add two large items $a_{2 L+1}=\sum_{\ell=1}^{2 L} a_{\ell}$ and $a_{2 L+2}=2 \sum_{\ell=1}^{2 L} a_{\ell}+1$ and set $A^{\prime}=A+a_{2 L+1}$. Then a subset $S$ in the instance of EQUALCARDINALITYSUBSETSUm exists if and only if $\sum_{\ell \in S} a_{\ell}+a_{2 L+1}=A^{\prime}$. We now create an instance $\mathcal{H}$ of the highway problem as follows. For every $\ell=1, \ldots, 2 L$, we define $a_{\ell}^{\prime}:=a_{\ell}+a_{2 L}$, and we introduce a gadget. Every gadget $\ell$ consists of four items $i_{\ell, 1}, i_{\ell, 2}, i_{\ell, 3}, i_{\ell, 4}$. Furthermore, there are $4+8 L$ bids in every gadget $\ell$, one bid is on bundle $\left\{i_{\ell, 1}\right\}$ with valuation $2 M-\frac{1}{2} a_{\ell}^{\prime}$, one bid is on bundle $\left\{i_{\ell, 2}\right\}$ with valuation $a_{\ell}^{\prime}, 8 L$ bids are on bundle $\left\{i_{\ell, 2}, i_{\ell, 3}\right\}$ with valuation $2 M+\left(\frac{1}{2}+\frac{1}{8 L}\right) a_{\ell}^{\prime}$, one bid is on bundle $\left\{i_{\ell, 3}\right\}$ with valuation $2 M-\frac{1}{2} a_{\ell}^{\prime}$, and one bid is on bundle $\left\{i_{\ell, 4}\right\}$ with valuation $M$, where $M$ is a sufficiently large integer. For gadget $2 L$, there is one additional bid on bundle $\left\{i_{2 L, 1}, i_{2 L, 2}, i_{2 L, 3}, i_{2 L, 4}\right\}$ with valuation $5 M$. Finally, there is one big bid on all items with valuation $10 M L+\frac{1}{8} a_{2 L}+\frac{1}{8 L} A$. Thus, the instance of the highway pricing problem has $2 L(4+8 L)+2$ bids and $8 L$ items, see Figure 1 . 


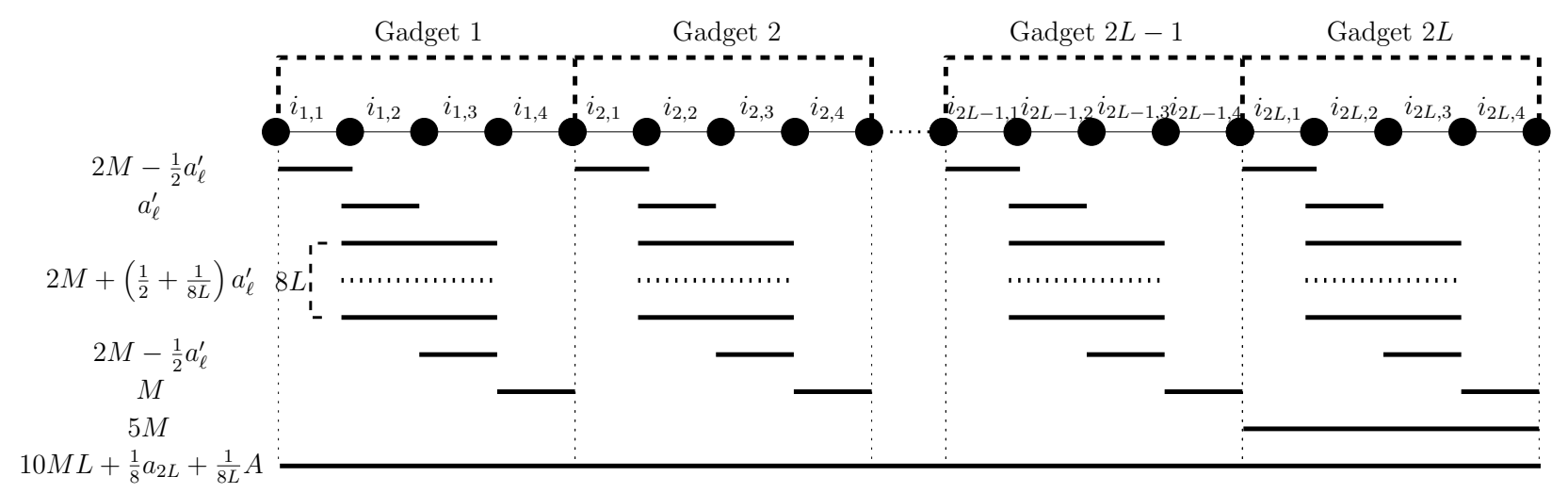

Fig. 1. Instance $\mathcal{H}$ of the highway problem.

To prove NP-hardness, we claim that there exists a set $S \subseteq\{1, \ldots, 2 L\}$ such that $\sum_{\ell \in S} a_{\ell}=A$ and $|S|=L$ if and only if there is a feasible solution for the highway problem, fulfilling the monotonicity constraint, and with a total revenue $\pi \geq(20+32 L) M L+5 M+4 L \sum_{\ell=1}^{2 L} a_{\ell}+\left(8 L^{2}+\frac{1}{8}\right) a_{2 L}+\frac{1}{8 L} A$. In the NP-hardness proof of Theorem 10 below, we use for every gadget only one out of two following price vectors.

$$
\begin{aligned}
& p_{\ell}^{1}=\left(2 M-\frac{1}{2} a_{\ell}^{\prime}, a_{\ell}^{\prime}, 2 M-\frac{1}{2} a_{\ell}^{\prime}, M\right) \\
& p_{\ell}^{2}=\left(2 M-\frac{1}{2} a_{\ell}^{\prime},\left(1+\frac{1}{8 L}\right) a_{\ell}^{\prime}, 2 M-\frac{1}{2} a_{\ell}^{\prime}, M\right) .
\end{aligned}
$$

Proposition 8 Suppose that there exists a solution to $\mathcal{H}$ with revenue at least $(20+32 L) M L+5 M+$ $4 L \sum_{\ell=1}^{2 L} a_{\ell}+\left(8 L^{2}+\frac{1}{8}\right) a_{2 L}+\frac{1}{8 L} A$. Then, the items in gadget $\ell$ are priced according to either price vector $p_{\ell}^{1}$ or $p_{\ell}^{2}$.

PROOF. Let us denote the total revenue by $\pi=\pi_{b i g}+\sum_{\ell=1}^{2 L} \pi_{\ell}$, where $\pi_{b i g}$ is the revenue obtained from the big bid and $\pi_{\ell}$ is the revenue obtained from the items in gadget $\ell$.

Consider a gadget $1 \leq \ell<2 L$. If $p_{\ell, 2}+p_{\ell, 3}>2 M+\frac{1}{2} a_{\ell}^{\prime}$, then at most one of the bids on $\left\{i_{\ell, 2}\right\}$ or $\left\{i_{\ell, 3}\right\}$ belongs to the set of winning bids. In this case, we can bound the revenue on gadget $\ell$ from above by $\pi_{\ell} \leq 2 M-\frac{1}{2} a_{\ell}^{\prime}+2 M-\frac{1}{2} a_{\ell}^{\prime}+8 L\left(2 M+\left(\frac{1}{2}+\frac{1}{8 L}\right) a_{\ell}^{\prime}\right)+M=5 M+\left(16 M+4 a_{\ell}^{\prime}\right) L$. On the other hand, if the bid on $\left\{i_{\ell, 2}\right\}$ and the one on $\left\{i_{\ell, 3}\right\}$ are both winning bids, then each of the bids on $\left\{i_{\ell, 2}, i_{\ell, 3}\right\}$ cannot contribute more than $2 M+\frac{1}{2} a_{\ell}^{\prime}$ to the revenue. Therefore, also in this case the revenue due to the items in this gadget can be bounded by $\pi_{\ell} \leq 5 M+\left(16 M+4 a_{\ell}^{\prime}\right) L$.

With similar arguments, we can bound the revenue on gadget $2 L$ by $\pi_{2 L} \leq 10 M+\left(16 M+4 a_{\ell}^{\prime}\right) L$, and the big bid contributes at most $10 M L+(1 / 8) a_{2 L}+(1 / 8 L) A$. Hence, the total revenue $\pi=\pi_{b i g}+\sum_{\ell=1}^{2 L} \pi_{\ell} \leq$ $10 M L+\frac{1}{8} a_{2 L}+\frac{1}{8 L} A+\sum_{\ell=1}^{2 L}\left(5 M+\left(16 M+4 a_{\ell}^{\prime}\right) L\right)+5 M \leq(20+32 L) M L+5 M+4 L \sum_{\ell=1}^{2 L} a_{\ell}+\left(8 L^{2}+\frac{1}{8}\right) a_{2 L}+$ $\frac{1}{8 L} A$. Therefore, to have a revenue of at least $(20+32 L) M L+5 M+4 L \sum_{\ell=1}^{2 L} a_{\ell}+\left(8 L^{2}+\frac{1}{8}\right) a_{2 L}+\frac{1}{8 L} A$, the revenues on each of the gadgets and the big bid need to be equal to the before mentioned upper bounds. It is easy to verify that only the price vectors $p_{\ell}^{1}$ and $p_{\ell}^{2}$ satisfy this constraint.

In the NP-hardness proof of Theorem 10 below, we use only the price vectors $p_{\ell}^{1}$ and $p_{\ell}^{2}$ defined in (3). We first show that these price vectors always lead to a pricing strategy that satisfies the monotonicity constraint. Lemma 9 Any pricing strategy that only uses price vectors $p_{\ell}^{1}$ and $p_{\ell}^{2}$ for each gadget satisfies the monotonicity constraint.

PROOF. Assume that $0 \leq a_{1} \leq a_{2} \leq \ldots \leq a_{2 L}$. Notice that both $p_{\ell}^{1}$ and $p_{\ell}^{2}$ consist of 4 item prices, and the sum of the item prices in $p_{\ell}^{1}$ is $5 M$ and in $p_{\ell}^{2}$ it is $5 M+\frac{1}{8 L} a_{\ell}^{\prime}$. Now consider an arbitrary bundle of size $K$, where $K \leq n=8 L$. Since only prices $p_{\ell}^{1}$ and $p_{\ell}^{2}$ are used for the gadgets, depending on the value of $K$ 
mod 4, we can easily determine lower and upper bounds on the price of any bundle of size $K$. This is a tedious yet straightforward case analysis, and we give the resulting lower and upper bounds in Table 1.

Table 1

Smallest and largest bundle prices for bundles with size $K$.

\begin{tabular}{|l|l|l|}
\hline$K \equiv 0 \bmod 4$ & $L B=5 M \frac{K}{4}+\frac{1}{2} a_{1}^{\prime}-\frac{1}{2} a_{2 L}^{\prime}$ & $U B=5 M \frac{K}{4}-\frac{1}{2} a_{1}^{\prime}+\left(\frac{1}{2}+\frac{K}{4} \cdot \frac{1}{8 L}\right) a_{2 L}^{\prime}$ \\
\hline$K \equiv 1 \bmod 4$ & $L B=5 M\left\lfloor\frac{K}{4}\right\rfloor+a_{1}^{\prime}$ & $U B=5 M\left\lfloor\frac{K}{4}\right\rfloor+2 M-\frac{1}{2} a_{1}^{\prime}+\left\lfloor\frac{K}{4}\right\rfloor \cdot \frac{1}{8 L} a_{2 L}^{\prime}$ \\
\hline$K \equiv 2 \bmod 4$ & $L B=5 M\left\lfloor\frac{K}{4}\right\rfloor+2 M+\frac{1}{2} a_{1}^{\prime}$ & $U B=5 M\left\lfloor\frac{K}{4}\right\rfloor+3 M-\frac{1}{2} a_{1}^{\prime}+\left\lfloor\frac{K}{4}\right\rfloor \cdot \frac{1}{8 L} a_{2 L}^{\prime}$ \\
\hline$K \equiv 3 \bmod 4$ & $L B=5 M\left\lfloor\frac{K}{4}\right\rfloor+3 M+\frac{1}{2} a_{1}^{\prime}$ & $U B=5 M\left\lfloor\frac{K}{4}\right\rfloor+5 M-a_{1}^{\prime}+\left\lfloor\frac{K}{4}\right\rfloor \cdot \frac{1}{8 L} a_{2 L}^{\prime}$ \\
\hline
\end{tabular}

One can now calculate the difference between the lower bound of a bundle of size $K+1$ and the upper bound on a bundle of size $K, L B(K+1)-U B(K)$. Recalling that $K \leq 8 L$ and $a_{\ell}^{\prime}=a_{\ell}+a_{2 L}$, one verifies that this difference is positive in all four cases, hence the monotonicity constraint is satisfied.

\section{Theorem 10 The highway problem with monotonicity constraint is NP-hard.}

PROOF. We show that there exists a set $S \subseteq\{1, \ldots, 2 L\}$ such that $\sum_{\ell \in S} a_{\ell}=A$ and $|S|=L$ if and only if there is a feasible solution for the highway problem, fulfilling the monotonicity constraint, and with a total revenue $\pi \geq(20+32 L) M L+5 M+4 L \sum_{\ell=1}^{2 L} a_{\ell}+\left(8 L^{2}+\frac{1}{8}\right) a_{2 L}+\frac{1}{8 L} A$. Given a set $S \subseteq\{1, \ldots, 2 L\}$ such that $\sum_{\ell \in S} a_{\ell}=A$ and $|S|=L$. Let the price vector of gadget $\ell$ be $p_{\ell}^{1}$ if $\ell \notin S$, and $p_{\ell}^{2}$ if $\ell \in S$. The revenue of every gadget $\ell$ is $(5+16 L) M+4 L\left(a_{\ell}+a_{2 L}\right)$, independent of which price vector is used, and there is an additional revenue in gadget $2 L$ of $5 M$. Given the pricing strategy for all items, the big bid contributes $\sum_{\ell \in S}\left(5 M+\frac{1}{8 L}\left(a_{\ell}+a_{2 L}\right)\right)+\sum_{\ell \notin S}(5 M)=10 L M+\frac{1}{8} a_{2 L}+\frac{1}{8 L} A$. This yields a total revenue of $(20+32 L) M L+5 M+4 L \sum_{\ell=1}^{2 L} a_{\ell}+\left(8 L^{2}+\frac{1}{8}\right) a_{2 L}+\frac{1}{8 L} A$.

For the converse, assume there is a feasible solution to the highway pricing problem with a total revenue of at least $(20+32 L) M L+5 M+4 L \sum_{\ell=1}^{2 L} a_{\ell}+\left(8 L^{2}+\frac{1}{8}\right) a_{2 L}+\frac{1}{8 L} A$. By Proposition 8 , we know that we use either $p_{\ell}^{1}$ or $p_{\ell}^{2}$ as defined in (3) for each gadget $\ell$. Notice that to obtain the maximum revenue on gadget $2 L$, we use pricing $p_{2 L}^{1}$. Let set $S=\left\{\ell: p_{\ell}=p_{\ell}^{2}\right\}$. Then, the actual payment of the big bid can also be written as $\sum_{\ell \in S}\left(5 M+\frac{1}{8 L}\left(a_{\ell}+a_{2 L}\right)\right)+\sum_{\ell \notin S}(5 M)=10 M L+\frac{1}{8 L}|S| a_{2 L}+\frac{1}{8 L} \sum_{\ell \in S} a_{\ell}$. From the arguments in the proof of Proposition 8, we know that this should be equal to its valuation $10 M L+\frac{1}{8} a_{2 L}+\frac{1}{8 L} A$. That is, $\sum_{\ell \in S} a_{\ell}=(L-|S|) a_{2 L}+A$. We claim that $|S|=L$. To prove this, suppose it is not true. First, assume that $|S|<L$, then $\sum_{\ell \in S} a_{\ell}=(L-|S|) a_{2 L}+A>\sum_{\ell=1}^{2 L-1} a_{\ell}+A$, which is not possible as $2 L \notin S$, so $|S| \geq L$. Now, assume $|S|>L$, then $\sum_{\ell \in S} a_{\ell}=(L-|S|) a_{2 L}+A<-a_{2 L}+A<0$, as $A<a_{2 L}$. This is also not possible as all integers $a_{\ell}$ are nonnegative. Therefore, we can conclude that $|S|=L$ and consequently, $A=\sum_{\ell \in S} a_{\ell}$.

\subsection{Approximation algorithm}

Notice that we cannot apply the PTAS from Theorem 7 to the highway problem, as this crucially requires the monotonicity constraint for arbitrary subsets of items. Nevertheless, we derive an $O(\log B)$ approximation algorithm for the highway pricing problem with monotonicity constraint, where $B=\max _{j} b_{j}$. To this end, we present approximation guarantees for two special cases first.

Lemma 11 The highway pricing problem with monotonicity constraint in which all bundles have size at least two is approximable within a factor of 3 by optimal uniform pricing.

PROOF. Consider an optimal solution with revenue OPT and let $p_{\max }^{*}$ be the highest item price in this solution. We claim that pricing all items at $p_{\max }^{*} / 3$, yields a revenue of at least OPT $/ 3$. Clearly, an optimal uniform pricing is at least as good as the uniform $p_{\max }^{*} / 3$ pricing.

First, we show that any winning bid $j \in W$ in the optimal pricing remains a winning bid for the uniform pricing at level $p_{\max }^{*} / 3$. Let $\left|I_{j}\right|=\ell$. Then the valuation for bid $j$ is at least $b_{j} \geq\lfloor\ell / 2\rfloor p_{\max }^{*}$, as by the monotonicity constraint the total price of any two consecutive items in an optimal solution is at least $p_{\text {max }}^{*}$ 
and the bidder who places bid $j$ can afford the corresponding bundle $I_{j}$. In the uniform $p_{\max }^{*} / 3$ pricing, the total bundle price is $\ell p_{\max }^{*} / 3$, which is at most $\lfloor\ell / 2\rfloor p_{\max }^{*}$, for $\ell \geq 2$. In an optimal pricing, bundle $I_{j}$ corresponding to bid $j$ is priced at most at $\ell p_{\max }^{*}$, whereas in our uniform pricing, we get $\ell p_{\max }^{*} / 3$. Hence, pricing all items $p_{\max }^{*} / 3$ yields a revenue of at least OPT $/ 3$.

The above lemma shows that whenever all bundles contain at least two items, we have a constant approximation. Now, we consider only instances in which bundles consist of exactly one item. Moreover, we restrict ourselves to instances in which $b_{j} / b_{k} \leq 2$, for any two bids $j$ and $k$.

Lemma 12 The highway pricing problem with monotonicity constraint, restricted to instances in which each bid is on a bundle with exactly one item and $b_{j} / b_{k} \leq 2$ for any two bids $j, k$, admits a linear time 2-approximation algorithm

PROOF. We price each item uniformly at $p=\min _{j} b_{j}$. Since for any two bids $j, k$, it holds that $b_{j} / b_{k} \leq 2$, and every bid is on a bundle with one item, we loose at most a factor of 2 for every bid. Hence, pricing all items at $p$ yields a revenue of at least OPT $/ 2$.

Theorem 13 The best uniform pricing yields a solution with revenue at least $\mathrm{OPT} /\left(3+2 \log _{2} B\right)$ for the highway problem with monotonicity constraint, where $B=\max _{j} b_{j}$. Moreover, the time needed to find this solution is $O\left(n^{2} m\right)$.

PROOF. Consider an optimum solution satisfying the monotonicity constraint, and let $\mathrm{OPT}_{L}$ denote the revenue of bidders whose bids are on bundles of size at least two and let $\mathrm{OPT}_{r}$ denote the revenue of bidders whose bids are on bundles of size one with valuation $2^{r-1} \leq b_{j}<2^{r}\left(r=1, \ldots,\left\lceil\log _{2} B\right\rceil+1\right)$ in this solution. Then $\mathrm{OPT}=\mathrm{OPT}_{L}+\sum_{r} \mathrm{OPT}_{r}$.

Moreover, let $\mathrm{APP}_{L}$ denote the revenue obtained by the best uniform pricing and $\mathrm{APP}_{r}$ denote the revenue obtained by the best uniform pricing strategy for the bidders with bids in $J_{r}=\left\{j \in J:\left|I_{j}\right|=1\right.$ and $2^{r-1} \leq$ $\left.b_{j}<2^{r}\right\}$. By Property 12 , we have that $\mathrm{APP}_{r} \geq \mathrm{OPT}_{r} / 2$ and thus $\max _{r} \mathrm{APP}_{r} \geq \sum_{r} \mathrm{OPT}_{r} /\left(2 \log _{2} B\right)$. Moreover, from Lemma 11, it follows that $\mathrm{APP}_{L} \geq \mathrm{OPT}_{L} / 3$. Hence, the solution found yields a revenue of $\max \left\{\mathrm{APP}_{L}, \mathrm{APP}_{r}: r=1, \ldots,\left\lceil\log _{2} B\right\rceil+1\right\} \geq \mathrm{OPT} /\left(3+2 \log _{2} B\right)$.

To see the claim on the time complexity, note that to find an optimal uniform pricing, we need to consider at most $\mathrm{O}(n)$ different prices, namely $b_{j} /\left|I_{j}\right|$ for all $j \in J$. For each price, we need to compute the set of winning bids and the revenue obtained on this price, which can be done in $\mathrm{O}(n m)$ time. So, the best uniform price can be computed in $\mathrm{O}\left(n^{2} m\right)$ time.

\section{Conclusion}

This paper studies purely algorithmic, or omniscient pricing problems, reflected by the fact that we assume bidders' valuations $b_{j}$ to be known. Even more challenging are problems where valuations are private information, and incentive-compatible (truthful) mechanisms are sought, that is, mechanisms that induce bidders to truthfully report their valuations. To that end, we refer to Goldberg et al. [9] or Balcan et al. [3], and note that Balcan et al. [4], extending corresponding results by Guruswami et al. [12], present a truthful mechanism with performance guarantee $O(\log n+\log m)$ for single item pricing in general combinatorial auctions with unlimited supply, hence particularly for the model considered in this paper. Again, the underlying pricing mechanism is very simple; it consists of randomly choosing a uniform price for all items. Thus, extending the approximation algorithms of this paper to truthful mechanisms is an interesting problem for future research.

\section{Acknowledgements}

Joyce van Loon acknowledges support by METEOR, the Maastricht Research School of Economics of Technology and Organizations. We thank the anonymous referee for careful reading and helpful remarks. 


\section{References}

[1] G. Aggarwal, T. Feder, R. Motwani, and A. Zhu, Algorithms for multi-product pricing, Automata, Languages and Programming - ICALP 2004 (J. Díaz, J. Karhumäki, A. Lepistö, and D. Sannella, eds.), Lecture Notes in Computer Science, vol. 3142, Springer, 2004, pp. 72-83.

[2] M. F. Balcan and A. Blum, Approximation algorithms and online mechanisms for item pricing, Proceedings of the 7th ACM Conference on Electronic Commerce, ACM, 2006, pp. 29-35.

[3] M. F. Balcan, A. Blum, J. D. Hartline, and Y. Mansour, Mechanism design via machine learning, Proceedings of the 46th IEEE Symposium on Foundations of Computer Science, FOCS, 2005, pp. 605-614.

[4] M. F. Balcan, A. Blum, and Y. Mansour, Improved guarantees for revenue maximization in combinatorial auctions, Working paper, July 2007.

[5] P. Briest and P. Krysta, Single-minded unlimited supply pricing on sparse instances, Proceedings of the 17th Annual ACM-SIAM Symposium on Discrete Algorithms, ACM-SIAM, 2006, pp. 1093-1102.

[6] E. D. Demaine, U. Feige, M.T. Hajiaghayi, and M. R. Salavatipour, Combination can be hard: Approximability of the unique coverage problem, Proceedings of the 17th Annual ACM-SIAM Symposium on Discrete Algorithms, ACM-SIAM, 2006, pp. 162-171.

[7] K. Elbassioni, R. Sitters, and Y. Zhang, A quasi-ptas for profit-maximizing pricing on line graphs, Proceedings of the 15th Annual European Symposium on Algorithms (L. Arge and E. Welzl, eds.), Lecture Notes in Computer Science, Springer, vol. 4698, 2007, pp.451-462.

[8] M. R. Garey and D. S. Johnson, Computers and intractability: A guide to the theory of np-completeness, W. H. Freeman, New York, 1979.

[9] A. V. Goldberg, J. D. Hartline, A. R. Karlin, M. Saks, and A. Wright, Competitive auctions, Games and Economic Behavior 55 (2006), 242-269.

[10] A. Grigoriev, J. van Loon, R. Sitters, and M. Uetz, How to sell a graph: Guidelines for graph retailers, Proceedings of the 32nd International Workshop on Graph-Theoretic Concepts in Computer Science (F. Fomin, ed.), Lecture Notes in Computer Science, vol. 4271, Springer, 2006, pp. 125-136.

[11] A. Grigoriev, J. van Loon, M. Sviridenko, M. Uetz, and T. Vredeveld, Bundle pricing with comparable items, Algorithms - ESA 2007 (L. Arge, M. Hoffmann, and E. Welzl, eds.), Lecture Notes in Computer Science, vol. 4698, Springer, 2007, pp. $475-486$.

[12] V. Guruswami, J. D. Hartline, A. R. Karlin, D. Kempe, C. Kenyon, and F. McSherry, On profit-maximizing envy-free pricing, Proceedings of the 16th Annual ACM-SIAM Symposium on Discrete Algorithms, ACM-SIAM, 2005, pp. 11641173.

[13] J. D. Hartline and V. Koltun, Near-optimal pricing in near-linear time, Algorithms and Data Structures - WADS 2005 (F. K. H. A. Dehne, A. López-Ortiz, and J.-R. Sack, eds.), Lecture Notes in Computer Sciences, vol. 3608, Springer, 2005, pp. $422-431$. 